\section{Chlorosulfonylated Calix[4]arenes: Precursors for Neutral Anion Receptors with a Selectivity for Hydrogen Sulfate}

Yury Morzherin, Dmitry M. Rudkevich, Willem Verboom, and David N. Reinhoudt*

Laboratory of Organic Chemistry, University of Twente, P.O. Box 217, 7500 AE Enschede, The Netherlands

Received July 12, 1993

\section{Introduction}

In nature phosphate and sulfate binding proteins are very important receptors for the active transport systems in the cell. ${ }^{1,2} \mathrm{~A}$ very high selectivity in binding has been observed in prokaryotic, periplasmic phosphate and sulfate binding proteins, which demonstrate $>10^{5}$ selectivity for binding phosphate over sulfate and sulfate over phosphate, respectively. ${ }^{3}$ In both proteins the specific binding exclusively takes place through hydrogen bonding.

Synthetic receptors that bind anions contain either positively charged guanidinium or ammonium groups ${ }^{4}$ or Lewis acid metal centers ${ }^{5}$ to accomplish anion binding. Recently we reported functionalized uranyl-containing salenes ${ }^{6}$ and sulfonamides ${ }^{7}$ derived from tris(aminoethyl)amine (TREN) that form complexes with hard anions in $\mathrm{CH}_{3} \mathrm{CN}$ with a selectivity for $\mathrm{H}_{2} \mathrm{PO}_{4}^{-}$. In the present paper we report anion receptors based on chlorosulfonylated calix [4]arenes. ${ }^{8}$

Calix[4]arenes are important building blocks in supramolecular chemistry. ${ }^{10,11}$ They can be (selectively) functionalized both at the phenolic $\mathrm{OH}$ groups (lower rim) and at the para positions of the phenol rings (upper rim). ${ }^{12}$

\section{Results and Discussion}

The starting calixarene tetraamides $1 \mathrm{~g}$ and $1 \mathrm{~h}$ were obtained by reaction of $1 \mathrm{a}$ with the appropriate $N, N$ -

(1) (a) Luecke, H.; Quiocho, F. A. Nature 1990, 347, 402. (b) He, J. J.; Quiocho, F. A. Science 1991, 251, 1479.

(2) Kanyo, Z. F.; Christianson, D. W. J. Biol. Chem. 1991, 266, 4264.

(3) Thatcher, G. R. J.; Cameron, D. R.; Nagelkerke, R.; Schmitke, J. J. Chem. Soc., Chem. Commun. 1992, 386 and refs cited therein.

(4) (a) Dietrich, B.; Guilhem, J.; Lehn, J.-M.; Pascard, C.; Sonveaux, E. Helv. Chim. Acta 1984, 67, 91. (b) Schmidtchen, F. P. J. Org. Chem 1986, 51, 5161. (c) Hosseini, M. W.; Blacker, A. J.; Lehn, J.-M. J. Am Chem. Soc. 1990, 112, 3896. (d) Deslongchamps, G.; Galán, A.; de Mendoza, J.; Rebek, Jr., J. Angew. Chem. Int. Ed. Engl, 1992, 31, 61. (e) Sessler, J. L.; Cyr, M.; Furuta, H.; Kral, V.; Mody, T.; Morishima, T.; Shionoya, M.; Weghorn, S. Pure Appl. Chem. 1993, 65, 393.

(5) (a) Blanda, M. T.; Horner, J. N.; Newcomb, M. J. Org. Chem. 1989, 54, 4626. (b) Reetz, M. T.; Niemeyer, C. M.; Harms, K. Angew. Chem. Int. Ed. Engl. 1991, 30, 1472. (c) Yang, X.; Knobler, C. B.; Hawthorne M. F. Ibid. 1991, 30, 1507. (d) Beer, P. D.; Hazlewood, C.; Hesek, D.; Hodacova, J.; Stokes, S. E.; J. Chem. Soc., Dalton Trans. 1993, 1327. (6) Rudkevich, D. M.; Stauthamer, W. P. R. V.; Verboom, W. Engbersen, J. F. J.; Harkema, S.; Reinhoudt, D. N. J. Am. Chem. Soc. 1992, 114, 9671 .

(7) Valiyaveettil, S.; Engbersen, J. F. J.; Verboom, W.; Reinhoudt, D. N. Angew. Chem. 1993, 105, 942.

(8) After completion of this work Beer et al. ${ }^{9}$ reported a lower rim functionalized calix [4]arene anion receptor.

(9) Beer, P. D.; Dickson, C. A. P.; Fletcher, N.; Goulden, A. J.; Grieve, A.; Hodacova, J.; Wear, T. J. Chem. Soc., Chem. Commun. 1993, 828 (10) Gutsche, C. D. Calixarenes; Monographs in supramolecular chemistry; Stoddart, J. F., Ed.; The Royal Society of Chemistry: Cambridge, 1989; Vol 1.

(11) Vicens, J., Bohmer, V., Eds. Calixarenes: a versatile class of macrocyclic compounds; Topics in inclusion science; Kluwer Academic Press: Dordrecht, 1991.

(12) Van Loon, J.-D.; Verboom, W.; Reinhoudt, D. N. Org. Prep. Proc. Int. 1992, 24, 437. dialkyl-2-chloroacetamide in the presence of potassium iodide and $\mathrm{K}_{2} \mathrm{CO}_{3}$ as a base in refluxing acetonitrile for 18 $\mathrm{h}$ in 78 and $58 \%$ yield, respectively. Reaction of calix[4] arenes 1a,c,e,f (all cone conformation) with 40 equiv of chlorosulfonic acid in $\mathrm{CHCl}_{3}$ at room temperature for 2-3 h (method A) afforded the tetrakis(chlorosulfonyl)-

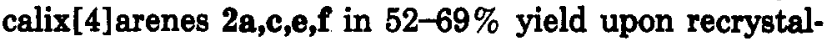
lization of the crude reaction mixture. The ${ }^{1} \mathbf{H}$ NMR spectra of $2 a, c, e, f$ indicate the presence of four identical rings. Under these conditions the tetrapropoxycalix [4]arene $1 \mathbf{b}$ did not give the expected tetrakis(chlorosulfonyl)calix [4] arene $2 b$ but the tetrahydroxytetrakis(chlorosulfonyl)calix [4] arene 2a in 35\% yield. Apparently under the acidic conditions, the propyl ether groups are not stable. ${ }^{13}$ Only a few examples of calixarenes having reactive chlorosulfonyl $\left(\mathrm{SO}_{2} \mathrm{Cl}\right)$ groups at the upper rim are known. ${ }^{15,16}$ They were prepared in two steps viz. sulfonylation followed by treatment with thionyl chloride, ${ }^{15,16}$ although it is known that the $\mathrm{SO}_{2} \mathrm{Cl}$ moiety can in principle be introduced in a one step $^{17}$ like in the synthesis of chlorosulfonyl benzocrown ethers. ${ }^{18}$

Under the same conditions, the 1,3-alternate conformer of 1c, calix [4]arene 1d, gave a complex reaction mixture. However, heating of $1 \mathrm{~d}$ at $50^{\circ} \mathrm{C}$ for $20 \mathrm{~min}(\operatorname{method} \mathrm{B})$ gave the tetrakis (chlorosulfonyl)calix [4] arene $2 \mathrm{~d}$ in $54 \%$ yield. The ${ }^{1} \mathrm{H}$ NMR spectrum of $2 \mathrm{~d}$ shows a singlet at $\delta$ 3.79 for the methylene bridge protons whereas in the ${ }^{13} \mathrm{C}$ NMR spectrum the corresponding carbon absorptions are present at $\delta 34.5$ which are both characteristic for a calix[4] arene in the 1,3-alternate conformation. ${ }^{19,20}$

Surprisingly, treatment of calix [4]arene amides $1 \mathrm{~g}$ and ih (cone conformation) with chlorosulfonic acid at room temperature for $2-3 \mathrm{~h}$ gave the bis(chlorosulfonyl)calix[4] arenes $3 a$ and $3 b$ in 42 and $27 \%$ yield, respectively. Probably under the strongly acidic conditions, the amide groups of $1 \mathrm{~g}$ and $1 \mathrm{~h}$ are protonated ${ }^{21}$ which results in a lower reactivity of the para positions of the corresponding aromatic rings. The two $\mathrm{SO}_{2} \mathrm{Cl}$ groups are introduced at diametrical aromatic rings as was concluded from the symmetry of the ${ }^{1} \mathrm{HNMR}$ spectra. The ${ }^{1} \mathrm{HNMR}$ spectrum of $3 \mathbf{a}$ exhibits a singlet at $\delta 7.79$ for the hydrogens of the chlorosulfonylated rings and a triplet and a doublet at $\delta$ 6.40 and 6.18 , respectively, for the hydrogens of the unreacted rings. Because of the symmetry there is only one $\mathrm{AB}$ system ( $\delta 5.34$ and 3.36) for the methylene bridge protons. Compounds $\mathbf{3 a}$ and $\mathbf{3 b}$ represent the first examples of calix [4] arenes having two $\mathrm{SO}_{2} \mathrm{Cl}$ groups. These compounds are not accessible via the two-step procedure because to the best of our knowledge selective sulfonylation

(13) Very recently Katritzky et al. ${ }^{14}$ reported the partial demethylation of 0 -nitroanisole upon reaction with chlorosulfonic acid.

(14) Katritzky, A. R.; Wu, J.; Rachwal, S.; Macomber, D.; Smith, T. P. Synth. Commun. 1993, 23, 405.

(15) Shinkai, S.; Kawaguchi, H.; Manabe, O. J. Polym. Sci., Polym. Lett. 1988, 26, 391.

(16) Shinkai, S.; Kawabata, H.; Matsuda, T.; Kawaguchi, H.; Manabe, O. Bull. Chem. Soc. Jpn. 1990, 63, 1272.

(17) Huntress, E. H.; Carten, F. H. J. Am. Chem. Soc. 1940, 62, 511.

(18) Markovsky, L. N.; Rudkevich, D. M.; Kalchenko, V. I. Zh. Org. Khim. 1989, 25, 1995. Chem. Abstr. 1990, 112, 216892w.

(19) Jaime, C.; de Mendoza, J.; Prados, P.; Nieto, P. M.; Sánchez, C. J. Org. Chem. 1991, 56, 3372.

(20) Verboom, W.; Datta, S.; Asfari, Z.; Harkema, S.; Reinhoudt, D. N. J. Org. Chem. 1992, 57, 5394.

(21) Homer, R. B.; Johnson, C. D. In The chemistry of functional groups. The chemistry of amides; Zabicky, J., Ed.; Interscience publishers: London, 1970; pp 188-197. 


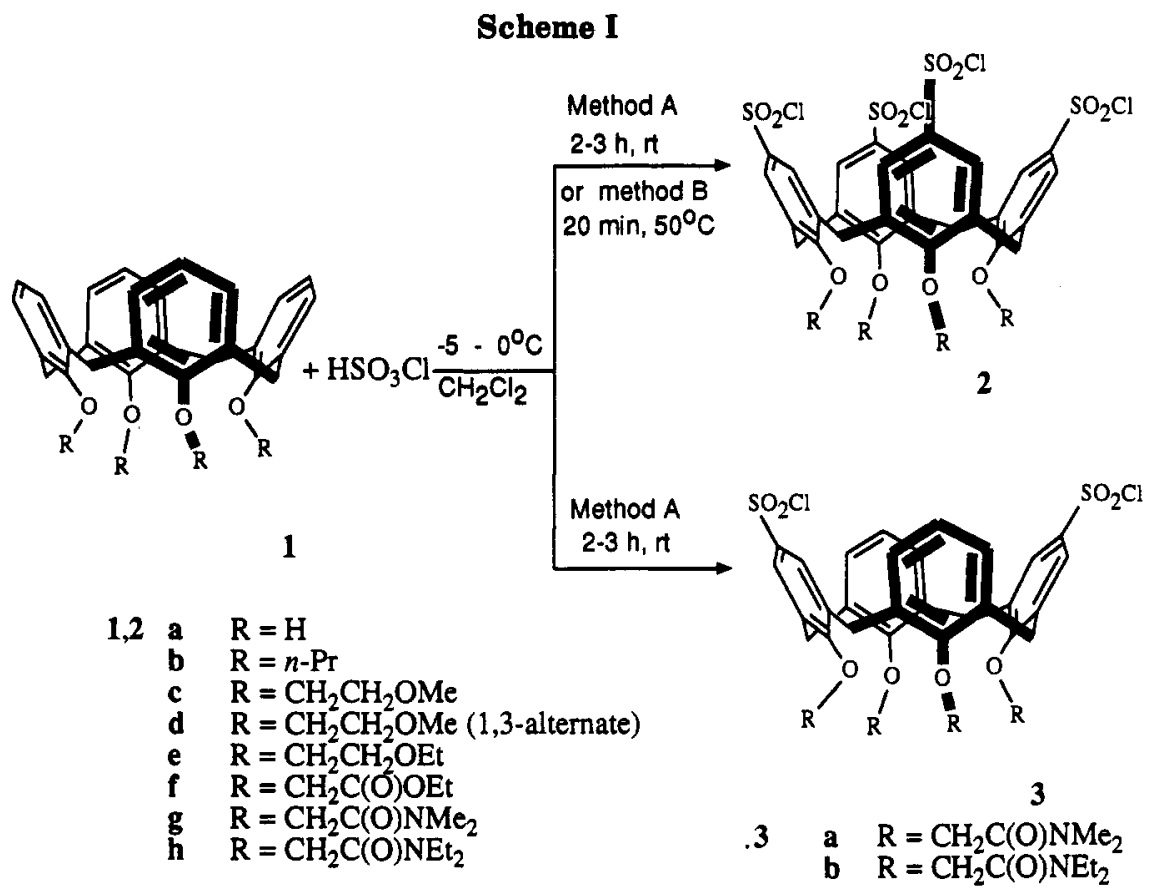

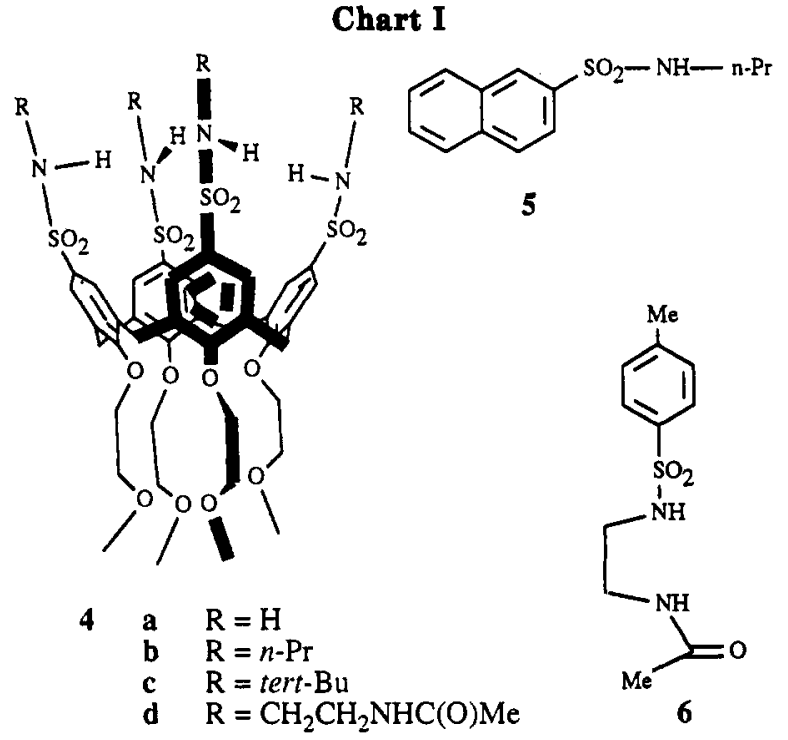

of calix [4] arenes is unknown. Method B gave the tetrakis(chlorosulfonyl)calix[4]arenes $\mathbf{2 g}$ and $2 \mathrm{~h}$ both in $61 \%$ yield.

Because TREN-derived sulfonamides form complexes with anions (vide supra), ${ }^{7}$ these tetrakis(chlorosulfonyl)calix[4]arenes 2 might be suitable precursors for the synthesis of hydrophobic, neutral anion receptors. Reaction of $2 c$ with ammonia, $n$-propylamine, tert-butylamine, or $\mathrm{N}$-acetylethylenediamine in $\mathrm{CH}_{2} \mathrm{Cl}_{2}$ for $4 \mathrm{~h}$ gave the corresponding calix[4]arene sulfonamides $4 \mathrm{a}-\mathrm{d}$ in yields of $64,87,88$, and $59 \%$, respectively. We also isolated the solid complex of $4 \mathrm{~d}$ with $\mathrm{Bu}_{4} \mathrm{NHSO}_{4}$, the formation of which was confirmed by a satisfactory elemental analysis. In the ${ }^{1} \mathrm{H}$ NMR spectrum the $\mathrm{NH}$ absorption has been shifted from $\delta 6.90$ (free ligand) to $\delta 7.75$ (complex). In the negative FAB mass spectrum of the solid complex, in addition to a peak of the free ligand, also signals of $\left[\mathrm{L}+\mathrm{HSO}_{4}{ }^{-}\right]-$and $\left[\mathrm{L}+\mathrm{Bu}_{4} \mathrm{NHSO}_{4}\right]-$ are present. The association constants $K$ of the 1:1 complexes of $\mathbf{4 b -}$
Table I. Association Constants $\left(K, \mathrm{M}^{-1}, \mathrm{CDCl}_{\mathbf{8}}\right)$ of Complexation of $4 b-d, 5$, and 6 with Different Anionsa

\begin{tabular}{crrrrr}
\hline & \multicolumn{5}{c}{ anions $^{b}$} \\
\cline { 2 - 6 } compd & $\mathrm{H}_{2} \mathrm{PO}_{4}^{-}$ & $\mathrm{HSO}_{4}^{-}$ & \multicolumn{1}{c}{$\mathrm{Cl}^{-}$} & $\mathrm{NO}_{\mathbf{3}^{-}}$ & $\mathrm{ClO}_{4}^{-}$ \\
\hline $\mathbf{4 b}$ & 350 & 970 & 360 & 240 & $<1$ \\
4c & $<10$ & 134 & 72 & 43 & $<1$ \\
4d & $c$ & 103400 & 1250 & 513 & $<1$ \\
5 & 14 & 10 & 15 & $<10$ & 16 \\
6 & 262 & 350 & 330 & 99 & 84
\end{tabular}

a The error is $<5 \%,{ }^{b}$ The counterion is $\mathrm{Bu}_{4} \mathrm{~N}^{+} .{ }^{c}$ Compound $4 \mathrm{~d}$ shows a complicated (mixed) complexation with $\mathrm{H}_{2} \mathrm{PO}_{4}-$ (compare refs 7 and 32); no $K$ value for 1:1 complexation could be determined.

$\mathrm{d}^{22}$ (and of reference compounds 5 and 6) with the tetrabutylammonium salts of $\mathrm{H}_{2} \mathrm{PO}_{4}^{-}, \mathrm{HSO}_{4}^{-}, \mathrm{Cl}^{-}, \mathrm{NO}_{3}^{-}$, and $\mathrm{ClO}_{4}^{-}$- in $\mathrm{CDCl}_{3}$ have been determined with ${ }^{1} \mathrm{H}$ NMR titration experiments and are summarized in Table I. Surprisingly in all cases a selectivity for $\mathrm{HSO}_{4}^{-}$was observed. The influence of the presence of four more or less preorganized binding sites is very clear comparing the $K$ values of $4 \mathbf{b}, \mathbf{c}$ and $4 \mathrm{~d}$ with those of the corresponding reference compounds 5 and 6, respectively. For all anions $4 d$ shows the highest $K$ values which may be due to the presence of four amide functions in addition to four sulfonamide moieties. However, more important is that $4 \mathrm{~d}$ exhibits for $\mathrm{HSO}_{4}^{-}$a selectivity of about $10^{2}$ over $\mathrm{Cl}^{-}$ and $\mathrm{NO}_{3}{ }^{-}$. Obviously the three-dimensional cavity of $4 \mathrm{~d}$ complexes the tetrahedral $\mathrm{HSO}_{4}$ - better than the spherical $\mathrm{Cl}^{-}$and the planar $\mathrm{NO}_{3}$-. To the best of our knowledge $4 b-d$ represent the first anion receptors with a selectivity for $\mathrm{HSO}_{4}-.^{23}$

\section{Experimental Section}

General. Melting points are uncorrected. ${ }^{1} \mathrm{H}$ and ${ }^{18} \mathrm{C}$ NMR spectra were recorded in $\mathrm{CDCl}_{3}$ with $\mathrm{Me}_{4} \mathrm{Si}$ as internal standard unless stated otherwise. Fast atom bombardment (FAB) mass spectra were obtained with $m$-nitrobenzyl alcohol as a matrix.

(22) Due to the presence of broad signals in the ${ }^{1} \mathrm{H}$ NMR spectrum of $4 \mathrm{a}$ in $\mathrm{CDCl}_{8}$ the anion complexation behavior has not been studied.

(23) Examples of nonselective (hydrogen) sulfate complezation have been reported in refs 4a, 6, 7, and Smith, P. J.; Reddington, M. V.; Wilcox, C. S. Tetrahedron Lett. 1992, 33, 6085 . 
Table II. Yields, Melting Points, and Characteristic Spectral Data of Compounds 2a,c-h*

\begin{tabular}{|c|c|c|c|c|c|c|c|c|}
\hline \multirow[b]{2}{*}{ compd } & \multirow[b]{2}{*}{$\begin{array}{l}\text { yield } \\
(\%)\end{array}$} & \multirow[b]{2}{*}{$\mathrm{mp}\left({ }^{\circ} \mathrm{C}\right)$} & \multicolumn{3}{|c|}{${ }^{1} \mathrm{H} \mathrm{NMR}\left(\mathrm{CDCl}_{3}\right)(\delta)$} & \multicolumn{2}{|c|}{${ }^{13} \mathrm{C} \mathrm{NMR}\left(\mathrm{CDCl}_{3}\right)(\delta)$} & \multirow{2}{*}{$\begin{array}{l}\text { FAB-MS } \\
m / z\left(\mathbf{M}^{+}\right) \\
\text {calcd }\end{array}$} \\
\hline & & & $\begin{array}{c}\text { ArH } \\
(\mathrm{s}, 8 \mathrm{H}) \\
\end{array}$ & $\mathrm{OCH}_{2}$ & $\mathrm{ArCH}_{2} \mathrm{Ar}$ & $\begin{array}{l}\mathrm{ArC}-\mathrm{SO}_{2} \\
(\mathrm{~s})\end{array}$ & $\begin{array}{l}\overline{\mathrm{ArCH}} \mathrm{H}_{2} \mathrm{Ar} \\
(\mathrm{t})\end{array}$ & \\
\hline $\begin{array}{l}2 a^{b} \\
2 c\end{array}$ & $\begin{array}{l}52^{\circ} \\
69\end{array}$ & $\begin{array}{r}>230 \text { dec } \\
172-174\end{array}$ & $\begin{array}{l}7.37 \\
7.50\end{array}$ & $\begin{array}{l}4.32(\mathrm{t}, 4 \mathrm{H}, J=4.4 \mathrm{~Hz}) \\
3.80(\mathrm{t}, 4 \mathrm{H}, J=4.4 \mathrm{~Hz})\end{array}$ & $\begin{array}{l}3.94(\mathrm{~s}, 8 \mathrm{H}) \\
4.74(\mathrm{~d}, 4 \mathrm{H}, J=13.5 \mathrm{~Hz}) \\
3.43(\mathrm{~d}, 4 \mathrm{H}, J=13.5 \mathrm{~Hz})\end{array}$ & $\begin{array}{l}138.0 \\
138.5\end{array}$ & $\begin{array}{l}30.4 \\
30.5\end{array}$ & $\begin{array}{c}817.0(817.5) \\
1015.3^{d}(1015.3)\end{array}$ \\
\hline 2d & 54 & $>290$ dec & 7.94 & $\begin{array}{l}4.01(\mathrm{t}, 4 \mathrm{H}, J=1.8 \mathrm{~Hz}) \\
3.77(\mathrm{t}, 4 \mathrm{H}, J=1.8 \mathrm{~Hz})\end{array}$ & $3.79(\mathrm{~s}, 8 \mathrm{H})$ & 138.1 & 34.5 & $1051.0^{\circ}(1050.9)$ \\
\hline $2 e$ & 53 & $212-213$ & 7.49 & $\begin{array}{l}4.35(\mathrm{t}, 4 \mathrm{H}, J=4.4 \mathrm{~Hz}) \\
\quad 3.80(\mathrm{t}, 4 \mathrm{H}, J=4.4 \mathrm{~Hz})\end{array}$ & $\begin{array}{l}4.77(\mathrm{~d}, 4 \mathrm{H}, J=13.7 \mathrm{~Hz}) \\
\quad 3.42(\mathrm{~d}, 4 \mathrm{H}, J=13.7 \mathrm{~Hz})\end{array}$ & 138.8 & 31.0 & $1070.9^{d}(1071.1)$ \\
\hline $2 f$ & 65 & $108-109$ & 7.53 & $4.88(\mathrm{~s}, 8 \mathrm{H})$ & $\begin{array}{c}5.14(\mathrm{~d}, 4 \mathrm{H}, J=14.0 \mathrm{~Hz}) \\
3.55(\mathrm{~d}, 4 \mathrm{H}, J=14.0 \mathrm{~Hz})\end{array}$ & 139.9 & 31.2 & $1162.9(1162.9)$ \\
\hline $2 g^{b}$ & 61 & $208-209$ & 7.21 & $5.02(8,8 \mathrm{H})$ & $\begin{array}{l}4.96(\mathrm{~d}, 4 \mathrm{H}, J=13.0 \mathrm{~Hz}) \\
3.48(\mathrm{~d}, 4 \mathrm{H}, J=13.0 \mathrm{~Hz})\end{array}$ & 140.0 & 31.9 & $1159.0(1158.9)$ \\
\hline $\mathbf{2 h}$ & 61 & $226-228$ & 7.50 & $5.15(8,8 \mathrm{H})$ & $\begin{array}{l}5.66(\mathrm{~d}, 4 \mathrm{H}, J=13.7 \mathrm{~Hz}) \\
3.48(\mathrm{~d}, 4 \mathrm{H}, J=13.7 \mathrm{~Hz})\end{array}$ & 139.1 & 32.6 & $1234.9^{d}(1235.6)$ \\
\hline
\end{tabular}

a All compounds gave satisfactory elemental analyses. ${ }^{b}$ In DMSO-d $d_{6 .}{ }^{c}$ Starting from $1 \mathrm{~b}$ the yield is $35 \% .{ }^{d}(\mathrm{M}-\mathrm{Cl})^{+} . e^{e}(\mathrm{M}-\mathrm{H})^{+}$.

$\mathrm{CH}_{2} \mathrm{Cl}_{2}$ was distilled from $\mathrm{CaH}_{2}$ and stored over molecular sieves. Calix [4] arenes $1 \mathrm{a},{ }^{24} 1 \mathrm{~b},{ }^{25} 1 \mathrm{c},{ }^{28} 1 \mathrm{~d},{ }^{20} 1 \mathrm{e},{ }^{27}$ and $1 \mathrm{f},{ }^{28}$ and reference compounds $6^{29}$ and $6^{30}$ were prepared according to literature procedures. All reactions were carried out under an argon atmosphere.

In the workup procedures the (combined) organic layers were washed with water (2x) and dried with $\mathrm{MgSO}_{4}$, whereupon the solvent was removed under reduced pressure. The presence of solvent in the analytical samples was confirmed by ${ }^{1} \mathrm{H}$ NMR spectroscopy.

General Procedure for the Preparation of $1 \mathrm{~g}, \mathrm{~h}$. A mixture of calix[4]arene 1a $(4.24 \mathrm{~g}, 0.01 \mathrm{~mol}), N, N$-dialkyl-2-chloroacetamide $(0.1 \mathrm{~mol})$, sodium iodide $(15 \mathrm{~g}, 0.1 \mathrm{~mol})$, and $\mathrm{K}_{2} \mathrm{CO}_{3}(13.8$ $\mathrm{g}, 0.1 \mathrm{~mol}$ ) in acetonitrile (100 mL) was refluxed for $18 \mathrm{~h}$. After filtration the solvent was removed. The residue was taken up in $\mathrm{CH}_{2} \mathrm{Cl}_{2}(150 \mathrm{~mL})$ and washed with water $(3 \times 400 \mathrm{~mL})$. Pure compounds were obtained upon recrystallization of the crude reaction products from $\mathrm{MeOH}$.

25,26,27,28-Tetrakis[(dimethylcarbamoyl)methoxy]calix[4]arene (1g): yield $78 \% ; \mathrm{mp} 256-258{ }^{\circ} \mathrm{C}$; ${ }^{1} \mathrm{H}$ NMR $\delta 6.7-6.5$ $(\mathrm{m}, 12 \mathrm{H}), 5.11$ (d, $4 \mathrm{H}, J=13.6 \mathrm{~Hz}), 4.84(\mathrm{~s}, 8 \mathrm{H}), 3.25(\mathrm{~d}, 4 \mathrm{H}$, $J=13.6 \mathrm{~Hz}), 3.00$ and $2.91(\mathrm{~s}, 2 \times 12 \mathrm{H}) ;{ }^{13} \mathrm{C}$ NMR $\delta 169.5(\mathrm{~s})$, 156.4 (s), 134.8 (s), 128.5 (d), 122.4 (d), 71.7 (t), 36.2 (q), 35.4 (q), 31.7 (t); MS-FAB $m / z$ 765.4 (M+ $\mathrm{M}^{+}$, calcd 764.9). Anal. Calcd for $\mathrm{C}_{4} \mathrm{H}_{62} \mathrm{~N}_{4} \mathrm{O}_{8}: \mathrm{C}, 69.09 ; \mathrm{H}, 6.85 ; \mathrm{N}, 7.32$. Found: $\mathrm{C}, 69.28 ; \mathrm{H}, 7.05$; $\mathrm{N}, 7.11$.

25,26,27,28-Tetrakis[(diethylcarbamoyl)methoxy]calix[4]arene (1h): yield $58 \% ; \mathrm{mp} 212-213^{\circ} \mathrm{C} ;{ }^{1} \mathrm{H}$ NMR $\delta 6.65-6.5(\mathrm{~m}$, $12 \mathrm{H}), 5.23$ (d, $4 \mathrm{H}, J=13.6 \mathrm{~Hz}), 4.90(\mathrm{~s}, 8 \mathrm{H}), 3.4-3.25(\mathrm{~m}, 16$ H), $3.23(\mathrm{~d}, 4 \mathrm{H}, J=13.6 \mathrm{~Hz}), 1.25-1.00(\mathrm{~m}, 24 \mathrm{H}) ;{ }^{13} \mathrm{C} \mathrm{NMR} \delta$ 168.6 (s), 156.6 (s), 134.9 (s), 128.4 (d), 122.2 (d), 71.5 (t), 40.9 (t), 39.9 (t), 31.9 (t), 14.3 (q) 13.1 (q); MS-FAB $m / z$ 877.4 (M+ ${ }^{+}$, calcd 877.1). Anal. Calcd for $\mathrm{C}_{62} \mathrm{H}_{68} \mathrm{~N}_{4} \mathrm{O}_{8}: \mathrm{C}, 71.21 ; \mathrm{H}, 7.81 ; \mathrm{N}, 6.39$. Found: C, 71.54; H, 8.02; N, 6.12.

General Procedure for the Preparation of Bis- and Tetrakis(chlorosulfonyl)calix [4]arenes 2 and 3. To a cooled solution of calix [4] arene $1(2 \mathrm{mmol})$ in $\mathrm{CHCl}_{3}(25 \mathrm{~mL})$ was added chlorosulfonic acid $(5.6 \mathrm{~mL}, 80 \mathrm{mmol})$ at a rate to keep the temperature between 0 and $10^{\circ} \mathrm{C}$. The reaction mixture was stirred at room temperature for 2-3 h (method A) or heated at $50^{\circ} \mathrm{C}$ for $20 \mathrm{~min}$ (method $\left.\mathrm{B}\right)$. The reaction mixture was poured onto ice $(100 \mathrm{~g})$ and extracted with $\mathrm{CH}_{2} \mathrm{Cl}_{2}(4 \times 50 \mathrm{~mL})$. The crude products were recrystallized from toluene to afford pure

(24) Gutsche, C. D.; Lin, L.-G. Tetrahedron 1986, 42, 1633.

(25) Imamoto, K.; Araki, K.; Shinkai, S. J. Org. Chem. 1991, 56, 4955. (26) Arduini, A.; Casnati, A.; Fabbi, M.; Minari, P.; Pochini, A.; Sicuri, A. R.; Ungaro, R. J. Supramol. Chem. 1993, 1, 235.

(27) Arduini, A.; Manfredi, G.; Pochini, A.; Sicuri, A.; Ungaro, R. J. Chem. Soc., Chem. Commun. 1991, 936.

(28) Arnaud-Neu, F.; Collins, E. M.; Deasy, M.; Ferguson, G.; Harris, S. J.; Kaitner, B.; Lough, A. J.; McKervey, M. A.; Marques, E.; Ruhl, B. L.; Schwing-Weill, M. J.; Seward, E. M. J. Am. Chem. Soc. 1989, 111, 8681.

(29) Campbell, K. N.; Campbell, B. K.; Salm, E. M. Proc. Indiana Acad. Sci. 1948, 57, 97.

(30) Mikiciuk-Olasik, E. Pol. J. Chem. 1989, 63, 279. compounds 2,3. The yields, melting points and selected spectral data of compounds 2 are summarized in Table II.

5,17-Bis(chlorosulfonyl)-25,26,27,28-tetrakis [(dimethylcarbamoyl)methoxy]calix[4]arene (3a): yield $42 \%$; mp 218-219 ${ }^{\circ} \mathrm{C} ;{ }^{1} \mathrm{H}$ NMR $\delta 7.79(\mathrm{~s}, 4 \mathrm{H}), 6.45-6.35(\mathrm{~m}, 2 \mathrm{H})$, 6.2-6.15 (m, $4 \mathrm{H}), 5.34(\mathrm{~s}, 4 \mathrm{H}), 4.59(\mathrm{~s}, 4 \mathrm{H}), 5.24$ and $3.36(\mathrm{~d}$, $2 \times 4 \mathrm{H}, J=13.8 \mathrm{~Hz}), 3.04(\mathrm{~s}, 6 \mathrm{H}), 2.96(\mathrm{~s}, 12 \mathrm{H}), 2.91(\mathrm{~s}, 6 \mathrm{H})$; ${ }^{13} \mathrm{C}$ NMR $\delta 169.2(\mathrm{~s}), 168.0$ (s), 163.7 (s), 155.3 (s), 137.9 (s), 137.1 (s), 132.0 (s), 128.3 (d), 127.9 (d), 123.7 (d), 72.2 (t), 71.9 (t), 36.0 (q), 35.7 (q), 35.5 (q), 35.4 (q), 31.7 (t); MS-FAB m/z 961.3 (M+ calcd 961.9). Anal. Calcd for $\mathrm{C}_{44} \mathrm{H}_{66} \mathrm{Cl}_{2} \mathrm{~N}_{4} \mathrm{O}_{12} \mathrm{~S}_{2} \cdot 1.2 \mathrm{CH}_{2} \mathrm{Cl}_{2}: \mathrm{C}$, 51.03; H, 4.96; N, 5.27. Found: C, 50.98; H, 5.15; N, 5.11.

5,17-Bis (chlorosulfonyl)-25,26,27,28-tetrakis [(diethylcarbamoyl)methoxy]calix [4]arene (3b): yield $37 \%$; mp 163-165 ${ }^{\circ} \mathrm{C} ;{ }^{1} \mathrm{H}$ NMR $\delta 7.79(\mathrm{~s}, 4 \mathrm{H}), 6.5-6.3(\mathrm{~m}, 2 \mathrm{H}), 6.3-6.2(\mathrm{~m}$, $2 \mathrm{H}), 5.35(\mathrm{~s}, 4 \mathrm{H}), 4.68(\mathrm{~s}, 4 \mathrm{H}), 5.38$ and $3.25(\mathrm{~d}, 2 \times 4 \mathrm{H}, J=$ $13.0 \mathrm{~Hz}), 3.5-3.0(\mathrm{~m}, 16 \mathrm{H}), 1.3-1.0(\mathrm{~m}, 24 \mathrm{H}) ;$ MS-FAB m/z 1073.4 [(M+H) $)^{+}$, calcd 1073.1]. Anal. Calcd for $\mathrm{C}_{52} \mathrm{H}_{66} \mathrm{Cl}_{2} \mathrm{~N}_{4} \mathrm{O}_{12} \mathrm{~S}_{2} \cdot 1.7 \mathrm{H}_{2} \mathrm{O}: \mathrm{C}, 56.43 ; \mathrm{H}, 6.33 ; \mathrm{N}, 5.07$. Found: $\mathrm{C}$, $56.10 ; \mathrm{H}, 6.13 ; \mathrm{N}, 4.83$. Karl Fisher titration calcd for $2 \mathrm{H}_{2} \mathrm{O}$ : 2.75. Found: 2.66 .

General Procedure for the Preparation of $4 \mathrm{a}-\mathrm{d}$. To a solution of $2 \mathrm{c}(1.05 \mathrm{~g}, 1 \mathrm{mmol})$ in $\mathrm{CH}_{2} \mathrm{Cl}_{2}(40 \mathrm{~mL})$ was added the appropriate alkylamine ( $10 \mathrm{mmol})$. In the case of $4 \mathrm{a}$ ammonia was bubbled through the solution for $10 \mathrm{~min}$. The reaction mixture was stirred at $\mathrm{rt}$ for $4 \mathrm{~h}$ and subsequently washed with $1 \mathrm{~N} \mathrm{HCl}(2 \times 50 \mathrm{~mL})$ and water $(3 \times 50 \mathrm{~mL})$. The crude reaction products were recrystallized from $\mathrm{MeOH}$ to give pure $4 \mathrm{a}-\mathrm{d}$.

25,26,27,28-Tetrakis(methoxyethoxy)-5,11,17,23-tetrakis(sulfamoyl)calix[4]arene (4a): yield $64 \% ; \mathrm{mp} 171-173^{\circ} \mathrm{C}$; ${ }^{1} \mathrm{H}$ NMR (DMSO- $\left.d_{\theta}\right) \delta 7.31(\mathrm{~s}, 8 \mathrm{H}), 6.96(\mathrm{~s}, 8 \mathrm{H}), 4.16$ and 3.79 $(t, 2 \times 8 \mathrm{H}, J=5.0 \mathrm{~Hz}), 4.53$ and $3.43(\mathrm{~d}, 2 \times 8 \mathrm{H}, J=13.3 \mathrm{~Hz})$, $3.34(\mathrm{~s}, 12 \mathrm{H}) ;{ }^{13} \mathrm{C}$ NMR (DMSO-d $d_{8} \delta 158.6(\mathrm{~s}), 138.0(\mathrm{~s}), 134.6$ (s), 125.9 (d), 73.6 (t), 71.2 (t), 58.0 (q), 30.1 (t); MS-FAB m/z 973.2 (M+; calcd 973.1). Anal. Calcd for $\mathrm{C}_{40} \mathrm{H}_{62} \mathrm{~N}_{4} \mathrm{O}_{16} \mathrm{~S}_{4} \cdot 1.5 \mathrm{H}_{2} \mathrm{O}$ : C, 48.04; H, 5.54; N, 5.60; S, 12.82. Found: C, 47.88; H, 5.80; N, 5.42; S, 13.01. Karl Fisher titration calcd for $1.5 \mathrm{H}_{2} \mathrm{O}: 2.70$. Found: 2.66 .

25,26,27,28-Tetrakis(methoxyethoxy)-5,11,17,23-tetrakis(propylsulfamoyl)calix[4]arene (4b): yield $87 \%$; mp 191$192^{\circ} \mathrm{C} ;{ }^{1} \mathrm{H}$ NMR $\delta 7.26(8,8 \mathrm{H}), 4.62$ and $3.30(\mathrm{~d}, 2 \times 4 \mathrm{H}, J=$ $13.4 \mathrm{~Hz}), 4.84(\mathrm{t}, 4 \mathrm{H}, J=6.2 \mathrm{~Hz}), 4.21$ and $3.79(\mathrm{t}, 2 \times 8 \mathrm{H}, J$ $=5.1 \mathrm{~Hz}$ ), $3.38(\mathrm{~s}, 12 \mathrm{H}), 2.86(\mathrm{q}, 8 \mathrm{H}, J=6.2 \mathrm{~Hz}$ ), 1.51 (sextet, $8 \mathrm{H}, J=6.2 \mathrm{~Hz}), 0.89(\mathrm{t}, 12 \mathrm{H}, J=6.2 \mathrm{~Hz}) ;{ }^{18} \mathrm{C} \mathrm{NMR} \delta 159.1(\mathrm{~s})$, $135.2(\mathrm{~s}), 135.1$ (s), $127.2(\mathrm{~d}), 73.8(\mathrm{t}), 71.6(\mathrm{t}), 58.6(\mathrm{q}), 45.1(\mathrm{t})$, 30.7 (t), 23.3 (t) 11.1 (q); MS-FAB $m / 21141.4\left(\mathrm{M}^{+}\right.$; calcd 1141.5). Anal. Calcd for $\mathrm{C}_{52} \mathrm{H}_{76} \mathrm{~N}_{4} \mathrm{O}_{18} \mathrm{~S}_{4} \cdot 0.7 \mathrm{H}_{2} \mathrm{O}: \mathrm{C}, 54.12 ; \mathrm{H}, 6.76 ; \mathrm{N}, 4.85$. Found: C, 53.99; H, 6.75; N, 4.83. Karl Fisher titration calcd for $0.7 \mathrm{H}_{2} \mathrm{O}: 1.09$. Found: 1.05 .

5,11,17,23-Tetrakis[(1,1-dimethylethyl)sulfamoyl)-25,26,27,28-tetrakis (methoxyethoxy) calix [4]arene (4c): yield 88\%; $\operatorname{mp~} 129-130^{\circ} \mathrm{C} ;{ }^{1} \mathrm{H}$ NMR $\delta 7.31(\mathrm{~s}, 8 \mathrm{H}), 4.73(\mathrm{~s}, 4 \mathrm{H}), 4.59$ and $3.29(\mathrm{~d}, 2 \times 4 \mathrm{H}, J=13.6 \mathrm{~Hz}), 4.19$ and $3.76(\mathrm{t}, 2 \times 8 \mathrm{H}, J=4.6$ $\mathrm{Hz}), 3.35$ (s, $12 \mathrm{H}), 1.19(\mathrm{~s}, 36 \mathrm{H}) ;{ }^{13} \mathrm{C}$ NMR $\delta 159.0$ (s), 138.0 (s), 134.9 (s), 127.4 (d), 73.6 (t), 71.5 (t), 58.6 (q), 54.9 (s), 30.2 (q); MS-FAB $m / z$ 1194.5 [(M-H)-, calcd 1195.2]. Anal. Calcd for 
$\mathrm{C}_{68} \mathrm{H}_{84} \mathrm{~N}_{4} \mathrm{O}_{10 \mathrm{~S}} \mathrm{~S}_{4} \cdot 0.7 \mathrm{H}_{2} \mathrm{O} ; \mathrm{C}, 55.58 ; \mathrm{H}, 7.11 ; \mathrm{N}, 4.63 ; \mathrm{S}, 10.60$. Found: C, 55.41; H, 7.28; N, 4.60; S, 10.53. Karl Fisher titration calcd for $0.7 \mathrm{H}_{2} \mathrm{O}: 1.04$. Found: 1.02 .

5,11,17,23-Tetrakis[[(2-acetylamino)ethyl]sulfamoyl]-25,26,27,28-tetrakis(methoxyethoxy)calix[4]arene (4d): yield $59 \% ; m p ~ 89-90{ }^{\circ} \mathrm{C} ;{ }^{1} \mathrm{H}$ NMR $\delta 7.25(\mathrm{~s}, 8 \mathrm{H}), 6.90(\mathrm{~s}, 4 \mathrm{H}), 6.30$ $(\mathrm{s}, 4 \mathrm{H}), 4.61$ and $3.32(\mathrm{~d}, 2 \times 4 \mathrm{H}, J=13.3 \mathrm{~Hz}), 4.25-4.15(\mathrm{~m}$, $8 \mathrm{H}), 3.8-3.75(\mathrm{~m}, 8 \mathrm{H}), 3.38(\mathrm{~s}, 12 \mathrm{H}), 3.35-3.25(\mathrm{~m}, 8 \mathrm{H}), 3.0-2.9$ $(\mathrm{m}, 8 \mathrm{H}), 1.99(\mathrm{~s}, 12 \mathrm{H}) ;{ }^{13} \mathrm{C}$ NMR $\delta 159.4(\mathrm{~s}), 158.9(\mathrm{~s}), 134.8(\mathrm{~s})$, $134.6(\mathrm{~s}), 127.0(\mathrm{~d}), 73.7(\mathrm{t}), 71.5(\mathrm{t}), 58.5(\mathrm{q}), 45.1(\mathrm{t}), 41.9(\mathrm{t}), 29.9$ $(\mathrm{t}), 22.9$ (q); MS-FAB $m / z$ 1313.7 (M-, calcd 1313.6). Anal. Calcd for $\mathrm{C}_{60} \mathrm{H}_{80} \mathrm{~N}_{8} \mathrm{O}_{20} \mathrm{~S}_{4} \cdot 0.5 \mathrm{H}_{2} \mathrm{O}: \mathrm{C}, 50.86 ; \mathrm{H}, 6.17 ; \mathrm{N}, 8.47 ; \mathrm{S}, 9.70$. Found: C, 51.07; H, 5.98; N, 8.52: S, 9.89. Karl Fisher titration calcd for $0.5 \mathrm{H}_{2} \mathrm{O}: 0.71$. Found: 0.71 .

Solid Complex of $4 \mathrm{~d}$ and Bu,NHSO 4 . A mixture of $4 \mathrm{~d}$ (132 $\mathrm{mg}, 0.1 \mathrm{mmol}$ ) and $\mathrm{Bu}_{4} \mathrm{NHSO}_{4}(34 \mathrm{mg}, 0.1 \mathrm{mmol})$ in $\mathrm{CHCl}_{3}(20$ $\mathrm{mL}$ ) was stirred at rt for $18 \mathrm{~h}$. The solvent was removed and the resulting solid dried: $m p 45^{\circ} \mathrm{C} ;{ }^{1} \mathrm{H}$ NMR $\delta 7.75(\mathrm{~s}, 4 \mathrm{H}), 7.37$ (s, $8 \mathrm{H}), 4.56$ and $3.38(\mathrm{~d}, 2 \times 4 \mathrm{H}, J=13.1 \mathrm{~Hz}), 4.18(\mathrm{t}, 8 \mathrm{H}, J=$ $5.1 \mathrm{~Hz}), 3.79(\mathrm{t}, 8 \mathrm{H}, J=5.1 \mathrm{~Hz}), 3.38(\mathrm{~s}, 12 \mathrm{H}), 3.4-2.9(\mathrm{~m}, 24$
$\mathrm{H}), 1.95(\mathrm{~s}, 12 \mathrm{H}), 1.9-1.3(\mathrm{~m}, 16 \mathrm{H}), 1.00(\mathrm{t}, 12 \mathrm{H}, J=7.3 \mathrm{~Hz})$; MS-FAB $m / z$ 1311.3 [(L-2 H) - $2 \mathrm{H})^{-}$, calcd 1408.7], 1650.2 [(L + BuNHSO 4 - $\left.3 \mathrm{H}\right)^{-}$, calcd 1650.2 ]. Anal. Calcd for $\mathrm{C}_{72} \mathrm{H}_{117} \mathrm{~N}_{8} \mathrm{O}_{24} \mathrm{~S}_{6} \cdot 0.75 \mathrm{CHCl}_{8}$ : $\mathrm{C}, 50.14$; H, 6.76; N, 7.24. Found: C, 49.88; H, 7.07; N, 7.35.

Determination of Association Constants. The measurements were performed by ${ }^{1} \mathrm{H}$ NMR titration experiments in $\mathrm{CDCl}_{3}$ at $298 \mathrm{~K}$ using a constant host concentration of $4 \mathrm{mM}$ and a varying guest concentration of $0.3-30 \mathrm{mM}$. For each $K$ value determination 5-10 different guest concentrations were taken. As a probe the chemical shift of the $\mathrm{SO}_{2} \mathrm{NH}$ signal was used. The $K$ values were calculated by nonlinear regression as described in ref 31.

Acknowledgment. We gratefully acknowledge Nuffic for financial support of Y.M.

(31) De Boer, J. A. A.; Reinhoudt, D. N.; Harkema, S.; van Hummel, G. J.; de Jong, F. J. Am. Chem. Soc. 1982, 104, 4073.

(32) Flatt, L. S.; Lynch, V.; Anslyn, E. V. Tetrahedron Lett. 1992, 33, 785. 